\title{
Free-Standing $\mathrm{LiNi}_{0.5} \mathrm{Mn}_{1.5} \mathrm{O}_{4} /$ Carbon Nanofiber Network Film as Light-Weight and High-Power Cathode for Lithium Ion Batteries-Supporting Information
}

Xin Fang, ${ }^{\dagger}$ Mingyuan Ge, ${ }^{\dagger}$ Jiepeng Rong ${ }^{\dagger}$ and Chongwu Zhou ${ }^{*}{ }^{*}$

${ }^{\dagger}$ Mork Family Department of Chemical Engineering and Materials Science and ${ }^{\ddagger}$ Ming Hsieh Department of Electrical Engineering, University of Southern California, Los Angeles, California 90089, United States

Email address for corresponding author: chongwuz@usc.edu

Electrochemical test of pure carbon nanofiber network

Pure carbon nanofiber (CNF) network without $\mathrm{LiNi}_{0.5} \mathrm{Mn}_{1.5} \mathrm{O}_{4}$ was tested in the voltage window of 3.5-5 V with Li metal as counter electrode. All other conditions were kept the same as battery tests for $\mathrm{LiNi}_{0.5} \mathrm{Mn}_{1.5} \mathrm{O}_{4} / \mathrm{CNF}$ network electrodes and conventional electrodes. The specific capacity of CNF network is shown in Figure S1. To make a fair comparison between $\mathrm{LiNi}_{0.5} \mathrm{Mn}_{1.5} \mathrm{O}_{4} / \mathrm{CNF}$ network electrodes and conventional electrodes, we have subtracted the capacity contributed from CNFs when calculating specific capacities for all $\mathrm{LiNi}_{0.5} \mathrm{Mn}_{1.5} \mathrm{O}_{4} / \mathrm{CNF}$ network electrodes.

Figure S1 


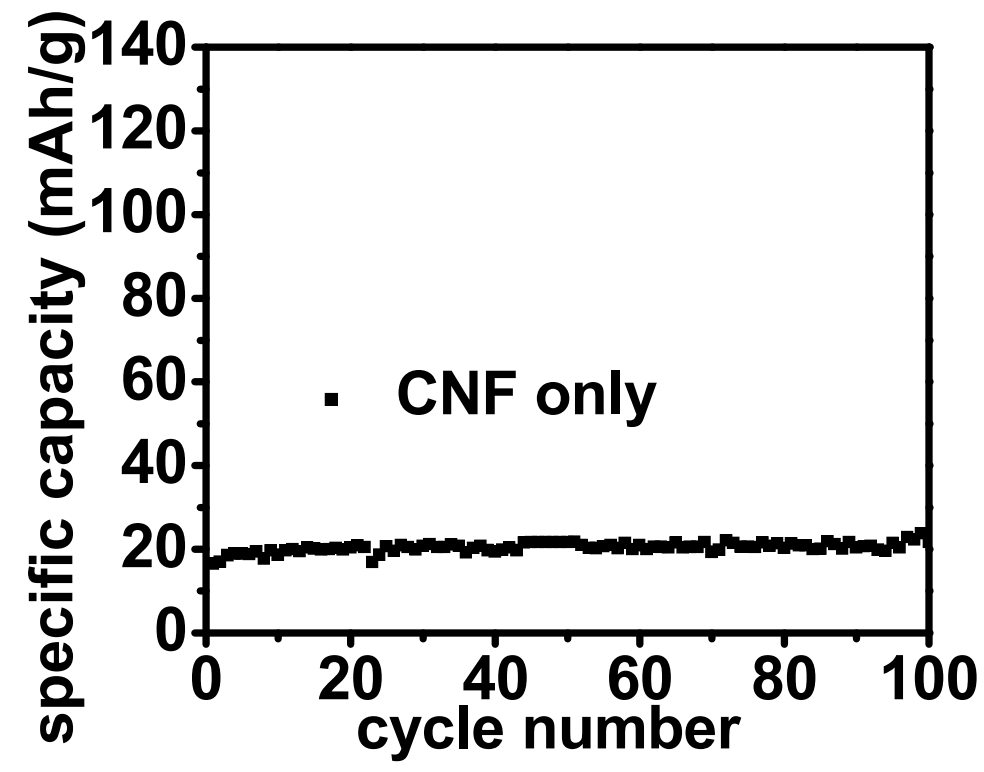

Figure S1. Specific capacity of carbon nanofibers at 3.5-5 V against Li metal. 